\title{
What Can We Learn from Cosmic Rays?
}

\section{S. Kövesi-Domokos* and G. Domokos}

Department of Physics and Astronomy

The Johns Hopkins University

E-mail: skd@jhu.edu

\begin{abstract}
Ultra high energy cosmic rays (UHECR) pose a problem either for particle physics or for astrophysics (or for both) by the unexpectedly high number of cosmic ray showers observed with energy above $\approx 5 \times 10^{19} \mathrm{eV}$, the Greisen-Zatsepin-Kuzmin (GZK) cutoff [1]. Our emphasis is on those possible solutions of the puzzle which assume that ultra high energy neutrinos travel cosmic distances. We present, in detail, a model which is based on a low energy (50 to $100 \mathrm{TeV}$ ) transition to a higher than four dimensional string regime. Neutrino-quark cross sections grow exponentially close to the threshold of this new scale because of the fast increase of the density of string states and effectively acquire hadronic strength.
\end{abstract}

\section{Introduction}

Cosmic rays were discovered about ninety years ago by Hess and Kohlhörster [2]. Through all these years they have provided a rich source of vital information on our galaxy and the universe beyond to astrophysics. Until the early nineteen fifties experimental particle physics could use only cosmic rays as the "incoming beam" for creating members of the particle zoo never seen before, like muons, pions, kaons, the lambda etc. However, the emerging accelerator physics with its well-controlled experimental environment put the interest in cosmic ray observations to the back burner as far as particle physics was concerned. During the last decade or so a dramatic turn-about took place. Atmospheric and solar neutrino detectors gave the first hint to the somewhat clueless particle physics community on the physics beyond the hugely successful, nevertheless incomplete Standard Model. Reactor and accelerator neutrino experiments gave confirmation of those cosmic ray results just last year. The limit on cosmic diffuse gamma radiation background by EGRET [3 combined with measurements of the cosmic ray spectrum at the highest energies provides restrictions on possible grand unified theories. The number of deeply penetrating horizontal showers measured by the grand array, AGASA [ [⿴囗十 and the nitrogen fluorescence detector Fly's

\footnotetext{
${ }^{*}$ Speaker.
} 
Eye [5] supplies bounds on larger than four dimensional models with low scale gravity [6. These are just some examples for the renewed interest in using observations concerning astrophysical processes and properties of the hot big bang cosmology as possible tests for predictions of theories going beyond the Standard Model [7].

\section{The significance of the high energy end of the spectrum}

A simplistic characterization of the measured cosmic ray spectrum affirms its isotropy, a composition, which is overwhelmingly hadronic (protons and nuclei) and a power law $\left(\propto E^{-\alpha}\right)$ energy dependence through about ten orders of magnitude from $10^{10} \mathrm{eV}$ to $10^{20} \mathrm{eV}$. There are two energy regions, $\approx 4 \times 10^{15} \mathrm{eV}$ and $\approx 10^{18.5} \mathrm{eV}$ (named the "knee" and the "ankle", respectively) where $\alpha$ changes quite abruptly: $\alpha \approx 2.7$ for $E \lesssim 10^{15.5}$ $\mathrm{eV}, \alpha \approx 3.1$ for $10^{15.5} \mathrm{eV} \lesssim E \lesssim 10^{18.5} \mathrm{eV}$ and $\alpha \approx 2.8$. for $E \gtrsim 10^{18.5} \mathrm{eV}$. For more details see [8]. Although a considerable number of substantially improved old detectors as well as new ones will provide crucial experimental observations for the "knee area" in cosmic rays $[8]$, for $\mathrm{GeV}$ and $\mathrm{TeV}$ gamma-rays and - hopefully - for neutrinos in the near future, the present paper brings into focus only the ultra high energy region of the cosmic ray spectrum.

The CM energy per particle at the Tevatron and at the LHC (1 TeV and $7 \mathrm{TeV}$, respectively) fall between the knee and the ankle on the cosmic ray spectrum, corresponding to fixed target energies $\approx 10^{15} \mathrm{eV}$ and $\approx 10^{17} \mathrm{eV}$. The highest energy event ever recorded has energy $3 \times 10^{20} \mathrm{eV}$ (by the Fly's Eye detector [9]), which is equivalent to about $500 \mathrm{TeV}$ in the CM system; otherwise, its extensive air shower (EAS) fits the properties of a proton or maybe a nucleus induced shower [10]. Clearly, at the high energy end of the spectrum the primary and some of the secondary interactions at the top of the atmosphere involve processes never tested in accelerator experiments; moreover, the highest energy events certainly scrutinize physics beyond the electroweak scale.

In addition, the region above the ankle presented the GZK puzzle. Up to about $10^{19} \mathrm{eV}$ energy the universe is transparent for protons, although the interaction with the everywhere present regular and chaotic magnetic fields takes away the directional information pointing back to their source. However, above that energy protons produce $e^{+} e^{-}$pairs and more importantly pions ${ }^{1}$ on the cosmic microwave background (CMB) radiation . At the energy $10^{19.5} \mathrm{eV}$ the mean free path for protons is down to about 7-8 Mpc and at each collision they lose about $20-25 \%$ of their energy. Consequently, the expectation 四 was that there should be a cutoff and just under the cutoff a pileup (a bump on the spectrum) as a consequence of the pion photoproduction for the above threshold protons or nuclei [11. (Nuclei photodisintegrate on the $\mathrm{CMB}$ and photons are absorbed (through $e^{+} e^{-}$production) on the $\mathrm{CMB}$ and on the universal radio background.)

There are somewhat less than a hundred extensive air showers observed with energies above $10^{19.5} \mathrm{eV}$ and about ten events above $10^{20} \mathrm{eV}$. The arrival directions are compat-

\footnotetext{
${ }^{1}$ Around this energy and above it the effect of the deflection by the galactic and intergalactic magnetic fields is small enough that with reasonable certainty a "source box" of a few degrees can be defined even for charged incoming cosmic particles.
} 
ible with an isotropic distribution of sources. Wherever determination was possible, the properties of the primary particles starting the EAS were consistent with being hadrons.

There is considerable disagreement on the errors of EAS energy estimates, on the finer features of the spectrum, and on the statistical significance of the data among the collaborations [15, 16, 17, 9, 团, which provided the observations. The recent analysis of the data already on file, the improvement of statistics by the continued operation of AGASA and HiRes, and by the future observations of new detectors (under construction like the Pierre Auger Observatory [18], and the planned Owl/Airwatch [19]) the reliability of the observational data will substantially improve in the next couple of years.

The propagation of nucleons, nuclei and gamma rays was studied in detail [12] with the conclusion that their sources cannot be very distant (at most $\approx 50-70 \mathrm{Mpc}$ away) ${ }^{2}$. There are only a few astrophysical sources, which can possibly accelerate particles to these extreme energies (e.g. AGN-s, radio galaxies). Since few of these objects are in the "GZK sphere" and the requirement of isotropy of the incoming extreme energy cosmic rays (EECR) also presents a severe restriction on their origin, the community started looking for new explanations for the higher than expected number of particles at the very end of the spectrum.

\section{GZK evading messengers}

There are two large classes of models which can provide interpretation for the data, top down and bottom up models ${ }^{3}$.

Top down models [20, 21] all use physics beyond the Standard Model. Topological defects and long lived relic particles could be produced everywhere during phase transitions experienced by the Universe after the Big Bang. Through their collapse/decay they produce the small number of EECR (protons) and dominantly photons and neutrinos. Their major advantage is that they avoid the problems of long range propagation in the background photon "gas", as well as the difficulties of accelerating particles to extreme energies. The art in the construction of these models is to make the required flux of EECR without creating too large a contribution to the diffuse gamma ray background bounded by the results of the EGRET detector [3]. Horizontal EAS (zenith angle larger than $60^{\circ}$ ) give limits for the neutrino flux.

Bottom up scenarios all face the acceleration problem. However, if - as a first step one can assume that the sources can be outside of the GZK sphere $\left(R_{G Z K} \approx\right.$ couple of tens $\mathrm{Mpc}$ ), then the number of sites able to produce EECR increases considerably. Among Standard Model particles only the neutrino can handle cosmic distances without dramatic absorption ${ }^{4}$. Both the Z-burst model and models inspired by string theory of $d>4$ use EECR neutrinos to increase the pool of sources.

\footnotetext{
${ }^{2}$ If the effect of the random extragalactic magnetic fields are taken into account, in addition to the energy loss processes, the average GZK distance of a proton further decreases [13] [14].

${ }^{3}$ For completeness, we must add the possibility of Lorentz symmetry violation, which could eliminate the GZK cutoff completely 20, 21].

${ }^{4}$ In principle, some supersymmetric particles can have their GZK cutoff at higher energies than the nucleons. However, LEP2 already excluded light superpartners. 20, 21]
} 
The "Z-burst" models [22] need no particle physics beyond the Standard Model (apart from non-zero neutrino masses) and assume standard hot Big Bang cosmology. They assert that extreme energy neutrinos $\left(\mathrm{E}=M_{Z}^{2} / 2 m_{\nu}\right)$ forming $\mathrm{Z}$ bosons resonantly with antineutrinos of the neutrino halo around our galaxy (or supergalaxy) could produce enough protons well inside the GZK radius to account for the EECR. The difficulties with this model were pointed out in ref. [12, 14]. Basically, the necessary requirements on the high energy neutrino flux and/or on the density of halo neutrinos make the scenario (nearly) incompatible with other astrophysical observations.

\section{String inspired models}

In all string inspired models the neutrino nucleon cross section is above the Standard Model value at very high energies. Internal consistency of string theories requires that strings live in a multidimensional space (typically, $\mathrm{d}=10$ for superstrings). It has been realized a few years ago that the connection between the string scale and the Planck scale is less rigid than hitherto believed [23].

In some models with large compactified extra dimensions, (or with a four-dimensional brane world) together with $\mathrm{TeV}$ scale quantum gravity [24 EECR neutrinos interact gravitationally with the nucleons in the atmosphere; as a matter of fact, that becomes the dominant interaction [25]. Unfortunately, the gravitational interaction does not give rise to a sufficiently rapidly growing cross section [26]. As a consequence, observational bounds on deep (nearly horizontal) showers are violated and it is not possible to reach cross sections comparable to hadronic ones around the GZK cutoff. The production of "mini black holes" has been also studied [26, 27]. Presently, it is unclear whether the upper bounds on deep showers are compatible with these theories.

At present, there is no internally consistent, phenomenologically viable string model known, in which even the basic features of the dynamics - including a mechanism of compactification - would be satisfactorily understood. Nevertheless, various string models have so many attractive properties that one is tempted to abstract their robust features and see whether some reasonable conjectures can be made once CMS energies of the colliding particles reach the string scale [14, 28]. For the sake of argument, let us have a string scale of the order of $50-100 \mathrm{TeV}$ in mind. This can be reached in ultra high energy cosmic ray interactions: for instance, the "gold plated" Fly's Eye event mentioned before has about $500 \mathrm{TeV}$ in the CMS. It was shown by means of an explicit calculation [29, 26] that weakly coupled string models cannot explain the trans-GZK cosmic ray interactions. Since we cannot calculate within the framework of a strongly coupled theory, we use features of current models, which are likely to be present in future, phenomenologically successful theories. The following basic ingredients are used:

- Unitarity of the $S$-matrix.

- A rapidly rising level density of resonances in dual models.

- Unification of interactions at around the string scale, hereafter denoted by $M$. 
- Duality between resonances in a given channel and Regge exchanges in crossed channels.

Since duality between resonances and Regge poles is exact only in the tree approximation to a string amplitude, it is unclear what the precise form of a generalization to world sheets of higher genus is: probably, resonances of finite width are dual to Regge cuts. Thus, our formulæ are likely to be valid to logarithmic accuracy. Using the optical theorem, the total cross section for the neutrino-parton interaction is ${ }^{5}$ :

$$
\hat{\sigma}(\hat{s})=\frac{8 \pi}{\hat{s}} \sum_{j}^{N(\hat{s})}(2 j+1)\left(1-\eta_{j} \cos \left(2 \delta_{j}\right)\right),
$$

where, as usual, $\eta$ and $\delta$ stand for the elasticity coefficient and phase shift of a given partial wave, respectively. The quantity $N(\hat{s})$ is the level of the resonance, equal to the maximal angular momentum. For elastic resonances, $\eta=1$ and $\delta \approx \pi / 2$ within the width of the resonance. Close to the threshold of the string regime, on resonance the total cross section is just proportional to the number of states at a given level. In any realistic model the resonances have finite widths, thus we average the cross section over an energy interval comparable to the widths of the resonances. In such an approximation, the density of states, $d(\hat{s})$ can be introduced and $N$ is regarded as a continuous variable, such that $N \approx \hat{s} / M^{6}$. Using this, one gets from eq. (4.1):

$$
\hat{\sigma} \approx \frac{16 \pi}{\hat{s}} d(\hat{s}) .
$$

As inelastic channels open up, the elasticity coefficients in eq. (4.1) become less than unity and eq. (4.2) is no longer valid. Without any detailed knowledge of the inelastic channels (world sheets of a higher genus in present day string models), we can estimate the behavior of the cross section as $\hat{s} \rightarrow \infty$ only. Duality tells us that the leptoquark excitations should be dual to the exchange of the $Z$-trajectory in the $t$-channel. Hence, apart from logarithmic corrections,

$$
\hat{\sigma} \sim \hat{s}^{(\alpha(0)-1)},
$$

where $\alpha(0)$ is the intercept (branch point, respectively) of the $Z$ trajectory. Apart from corrections of the order of $\left(M_{Z} / M\right)^{2}$, one has $\alpha(0)=1$, so that the neutrino-parton cross section tends to a constant. (We verify a posteriori that $M_{Z} / M \ll 1$, so that the power corrections to the cross section are insignificant at all energies of interest.)

The level density is a rapidly rising function of $\hat{s}$. It is known that asymptotically it rises as $\exp (a \sqrt{\hat{s} / M})$, with $a$ being some constant; see, for instance [30]. However, the rise is more rapid at the beginning of the spectrum. The first few levels of the open superstring can be well interpolated by the function

$$
d(N) \propto \exp 1.24 N, \quad N \approx \hat{s} / M,
$$

\footnotetext{
${ }^{5}$ All energies are assumed to be large compared to the rest energies of the incoming particles.

${ }^{6}$ In the last formula, the Regge intercept has been neglected. However, we shall see shortly that the excitations begin to contribute significantly to the cross section for $N \geq 10$ or so; hence this approximation is justified.
} 
(This approximate formula was calculated from the generating function of the level density, [30] eq. (4.3.64).) General considerations on a strongly coupled string model take us this far. To connect the low excitation regime, eq (4.2) and the asymptotic one, eq. (4.3) we chose an interpolating function guided by merely the requirement of simplicity. We found that after averaging over the parton distribution within the nucleon, the results are insensitive to the detailed form of the $\nu$-quark cross section. For that reason, we chose a simple form satisfying the limits at low and high excitations:

$$
\hat{\sigma}=\Theta\left(\hat{s}-M^{2}\right) \frac{16 \pi}{M^{2}} \frac{40 \exp 1.24 N_{0}}{1+\frac{\hat{s}}{M^{2}} \exp 1.24\left(N_{0}-\hat{s} / M^{2}\right)}
$$

In eq. (4.5), $M$ is the string scale and $N_{0}$ is a parameter measuring the onset of the "new physics". In fact, one can convert that dimensionless parameter into an energy scale. Using our previous relations, one can write $N_{0} \approx \hat{s_{0}} / M$, or in terms of a laboratory energy of the incoming neutrino, $N_{0} \approx 2 m \hat{E}_{0} / M, m$ being the mass of the nucleon. In all these equations, the "hat" over the energies involved serves as a reminder that the quantities have to be integrated over the parton distribution. As usual, the conversion is carried out by means of substitutions such as $\hat{s}=x s, x$ being the momentum fraction of a parton within the nucleon. The step function is inserted because the cross section of the "new physics" vanishes at CM energies below the mass of the first resonance. The parton distributions have been taken from CTEQ6 [31]. The dominant contribution comes from valence quarks; gluons do not contribute, since no presently known unification scheme contains "leptogluons". Finally, the contribution of the sea is negligibly small, since the latter is concentrated around $x=0$.

It is impossible to precisely determine the two parameters, $M$ and $N_{0}$ entering eq. (4.5). Nevertheless, the parameters can be bounded by the trans-GZK data. As it was mentioned before

- no deep showers have been observed by AGASA and Fly's Eye,

- the trans-GZK showers appear to be "hadron-like", i.e. they originate high in the atmosphere and appear to exhibit a development resembling proton induced showers.

These constraints were analysed [32]. The absence of deep showers excludes a region of the neutrino cross section, approximately, $0.02 \mathrm{mb} \leq \sigma \leq 1 \mathrm{mb}$. The cross section has to grow fast to roughly hadronic size around the "ankle" in the cosmic ray spectrum, approximately at $5 \times 10^{19} \mathrm{eV}$ and stay of this size or grow slightly. Unless these conditions are satisfied, the neutrino model of trans-GZK cosmic rays fails. A search of the parameter space yields $E_{0} \approx 5 \times 10^{10} \mathrm{GeV}$ and $M \approx 80 \mathrm{TeV}$. With these values of $M$ and $N_{0}$ the cross section is rising sufficiently rapidly to satisfy the deep shower bound and at the same time, it is sufficiently large in the trans-GZK energy region. These values of $E_{0}$ and $M$ give $N_{0} \approx 15.6$ confirming the intuitive expectation. The neutrino-nucleon cross section is shown in Fig. (四).

Due to the exponential dependence of eq. (4.5) on the parameters, one cannot vary their values over a broad range without getting a contradiction either with the bound on 


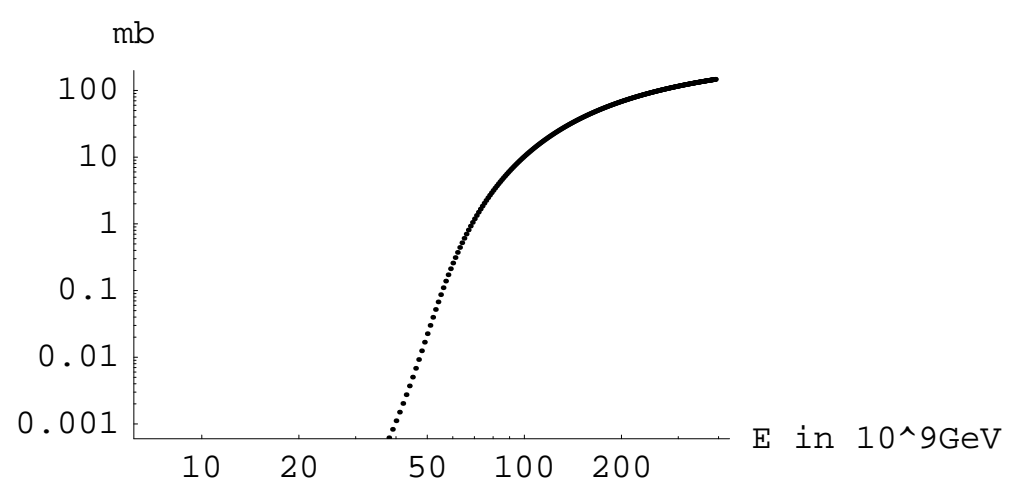

Figure 1: The $\nu$-nucleon cross section calculated from eq. (4.5) and the CTEQ6 parton distribution. $E_{0}=5 \times 10^{19} \mathrm{eV}, M=80 \mathrm{TeV}$.

deep showers and/or with the required value of the cross section for trans-GZK showers. Neutrino induced showers were simulated using the ALPS (Adaptive Longitudinal Profile Simulation) Monte Carlo package authored by Paul T. Mikulski[33]. Similarly to earlier studies, see, e.g. 34] it was assumed that quarks and leptons are created in comparable numbers in an interaction as long as the CM energy of an interaction remains above $M$. Once the energy drops below $M$, the usual Standard Model cross sections govern the further development of the shower. A qualitative consequence of this feature is that, statistically, neutrino induced showers exhibit larger fluctuations than proton induced ones, see $[34]^{7}$. In addition, once the cross section becomes larger than about 15-20 mb, the shower starts high in the atmosphere. Hence, on an event by event basis, such showers are virtually indistinguishable from hadron induced showers

\section{Conclusions}

The trans-GZK energy region presents a unique opportunity to get hints on the qualitative features of theories going beyond the Standard Model. In astrophysics, these extensive air showers may bring information either about cosmologically early times or about the most violent regions in the Universe. To be able to distinguish among these numerous imaginative scenarios, the collection of high statistics data is necessary. In the near future this will become possible with the continued operation and improvements of AGASA and HiRes and with the start of the hybrid (grand array and air fluorescence) megadetactor, the Pierre Auger Observatory.

\section{Acknowledgement}

We thank Professors K. Meier and O. Nachtmann for the organization and running of a

\footnotetext{
${ }^{7}$ This is a consequence of the fact that, once in the standard model regime, leptonic interactions have a lower average multiplicity than hadronic ones.
} 
very stimulating and inspiring Workshop.

\section{References}

[1] K. Greisen, Phys. Rev. Lett. 16,748 (1966); G.T. Zatsepin, and V.A. Kuzmin, Pisma Zh. Eksp. Theor. Fiz. 611028 (1966).

[2] V.F. Hess, Phys.Z. 13, 1084 (1912); W. Kohlhörster, Phys.Z. 14, 1153 (1913).

[3] P. Sreekumar, et al., Astrophys. J. 494, 523 (1990).

[4] AGASA Collaboration, H. Ohoka, S. Yoshida, and M. Takeda, Nucl. Instrum. Methods Phys. Res. A385, 268 (1997) and references quoted there.

[5] HIRES Collaboration, D.J. Bird et al., Astrophys. J. 424, 491 (1994).

[6] L.A. Anchordoqui, J.L. Feng, H. Goldberg, and A.D. Shapere, Phys.Rev. D 65, 124027 (2002); ibid. 66, 103002 (2002).

[7] G.G. Raffelt, Ann. Rev. Nucl. Part. Sci. 49, 163 (1999).

[8] H. Rebel, these Proceedings.

[9] D.J. Bird, et al., Phys. Rev. Lett. 71, 3401 (1993.)

[10] F. Halzen, R.A. Vazquez, T. Stanev, and H.P. Vankov, Astropart. Phys. 3, 151 (1995).

[11] C.T. Hill, D.N. Schramm, Phys. Rev. D31, 564 (1983).

[12] G. Sigl, in Physics and Astrophysics of Ultra-High-Energy Cosmic Rays, eds. M. Lemoine and G. Sigl (Springer, 2002).

[13] T. Stanev, et al. Phys. Rev. D62, 093005 (2000).

[14] G. Domokos, and S. Kövesi-Domokos, hep-ph/0107095 expanded version of a talk given at IECHEP2001, Budapest.

[15] Volcano Ranch: J. Linsley, Phys. Rev. Lett. 10, 146 (1963); M.T. Dova, et al. astro-ph/0210464.

[16] Haverah Park: M. Ave, et al. astro-ph/0112253

[17] Yakutsk: N.N. Efimov et al., in Astrophysical Aspects of the Most Energetic Cosmic Rays, eds. M.Nagano and F.Takahara (World Scientific, 1991).

[18] Pierre Auger Collaboration, Nucl. Phys. Proc. Suppl. 110, 487 (2002)

[19] J.F.Krizmanic et al., (OWL/AirWatch Collaboration), in Proceedings of the 26th International Cosmic Ray Conference, Salt Lake City 1999, Vol.2, 388-391.

[20] P. Bhattacharjee and G. Sigl in Physics and Astrophysics of Ultra-High-Energy Cosmic Rays, eds. M. Lemoine and G. Sigl (Springer, 2002).

[21] P. Bhattacharjee, and G. Sigl, Phys.Rep. 327, 109 (2000) and references quoted there.

[22] T.J. Weiler, Astropart. Phys. 11, 303 (1999); D. Fargion, B. Mele, A. Salis, Astrophys. J. 517, 725 (1999); Z. Fodor in these Proceedings.

[23] E. Witten, Nucl. Phys. B471, 135 (1996); J. Lykken, Phys. Rev. D54, 3693 (1996);

I. Antoniadis, S. Dimopoulos, and G. Dvali, Nucl. Phys. B516, 55 (1997). 
[24] N. Arkani-Hamed, S. Dimopoulos, and G. Dvali, Phys. Lett. B429, 263 (1998); I. Antoniadis, N. Arkani-Hamed, S. Dimopoulos, and G. Dvali, ibid. 436, 252 (1998); L. Randall and R. Sundrum, Phys. Rev. Lett. 83, 3370 (1999).

[25] N. Arkani-Hamed, et al., Phys. Rev. D59, 086004 (1999); S. Nussinov, and R. Shrock, Phys. Rev. D59, 105002 (1999); P. Jain, et al., Phys. Lett. B484, 267 (2000); J.P. Ralston, et al., in AIP Conf.Proc. 549, 733 (2002); M. Kachelriess and M. Plumacher, Phys. Rev. D62, 103006 (2000); L. Anchordoqui, et al., Phys.Rev. D63, 124009 (2001); S. Nussinov and R. Shrock, ibid. D64, 047702 (2001); M. Kachelriess and M. Plumacher, hep-ph/0109184.

[26] R. Emparan, M. Masip, and R. Rattazzi, Phys. Rev. D65, 064023 (2002).

[27] J.L. Feng and A.D. Shapere, Phys. Rev. Lett. 88, 021303 (2002).

[28] W.S. Burgett, et al., JHEP 0107:017 (2001), and hep-ph/0209162.

[29] F. Cornet, et al., Phys. Rev. Letters, 86, 4235 (2001).

[30] M.B. Green, J.H. Schwarz and E. Witten, "Superstring Theory". Cambridge University Press, Cambridge (1978). Chapter 5.

[31] J. Pumplin et al. www.phys.psu.edu/ cteq/

[32] G. Tyler, A.V. Olinto, and G. Sigl, Phys. Rev. D63, 055001 (2001); T.J. Weiler, in "Observing Ultrahigh Energy Cosmic Rays from Space and Earth", Edited by H. Salazar, L. Villaseñor and A. Zepeda. AIP Conference Proceedings, Vol. \# 566 (2001).

[33] Paul T. Mikulski, http://owl.gsfc.nasa.gov/mikulski-thesis.pdf

[34] G. Domokos et al., JHEP 07017 (2001). 